\title{
A hazard surgeons need to address
}

\author{
Peter T Scardino
}

Among the occupational hazards of being a surgeon, needle-stick injuries receive relatively little attention. Based on the statistics, we should be more concerned. Surgeons experience the most injuries from sharp objects of any healthcare worker. Most commonly, sharps injuries occur in the operating room (OR). A study of French surgeons estimated that the typical general surgeon experiences 0.8 injuries per $100 \mathrm{~h}$ of operating time, or 210 injuries throughout the course of a career, resulting in a $6.9 \%$ lifetime risk of contracting hepatitis C and a $0.15 \%$ lifetime risk of developing HIV. Even with widespread adoption of the Center for Disease Control's universal precautions engendered by the AIDS epidemic, the frequency of needle-stick injuries in the OR has remained constant over the last decade. Although the risk of contracting serious illness from needle-stick injuries is well known, surgeons comply poorly with the universal precautions, which might offer limited additional benefit over traditional aseptic techniques in the OR.

In addition to needle-stick injuries or scalpel wounds, which occur in 1.7-15\% of operations, members of the OR team are exposed to skin contamination from patients' blood in 6-50\% of cases. In the surgical team, surgeons sustain the highest risk of injury. Assistant surgeons and surgeons-in-training are close behind. Most of these injuries are self-inflicted and occur during suturing. Injuries are more common when procedures are complex, entail high blood loss, or involve multiple surgical teams and in a teaching environment. With needle injuries, transmission of the infective agent (seroconversion) can be expected in 10-30\% of health-care workers if the patient is infected with hepatitis $B, 1-10 \%$ with hepatitis $C$, and $0.1-0.3 \%$ with HIV.

The risk that a surgeon will acquire an active infection from an injury or exposure during an operation depends upon the severity of the injury, the type of needle involved (hollowbore needles are more likely to transmit infection than solid needles), the rate of infection
Until we

address

these issues, and make

compliance

easier... the

incidence of

sharps injuries

and resultant

serious illness

will remain

constant.

PT Scardino is Editorin-Chief of Nature

Clinical Practice

Urology.

\section{Competing interests}

The author declared he has no competing interests.

www.nature.com/clinicalpractice doi:10.1038/ncpuro0854 among the patient population, and the viral load of the patient's blood. The risk can be reduced substantially by relatively simple prophylactic measures, including double gloving (which reduces the frequency of skin exposure to blood by more than $85 \%$ and reduces blood on the needle by $95 \%$ ), immunization against hepatitis $\mathrm{B}$, and postexposure vaccination and/ or administration of immunoglobulin (for hepatitis B) or antiretroviral drugs (for HIV shortly after exposure). Surgeons generally comply poorly with these measures, except for hepatitis $B$ immunization, which is now required by most hospitals. Double gloving is not widely accepted, as many surgeons believe it impairs sensation and dexterity. Neutral zone procedures to minimize handing sharp instruments back and forth during an operation are rarely adopted despite formal recommendations from the American College of Surgeons and other professional bodies.

Perhaps the greatest impediment to reducing the rate of needle-stick injuries in the OR is that surgeons rarely report them. I asked our surgeons at a recent meeting who had not experienced a needle injury in the past year and none raised a hand. Serious barriers to reporting include the time required to provide a medical history and a blood sample at employee health, to obtain informed consent from the patient and order the necessary tests, and to return for the recommended follow-up visits. With an average exposure of over six needle-stick injuries a year for the typical surgeon, and skin contamination by blood in nearly $50 \%$ of surgical procedures, busy surgeons do not take the time to comply with the current regulations.

Until we address these issues, and make compliance easier-perhaps by bringing the employee health station into the OR suite regularly - the incidence of sharps injuries and resultant serious illness will remain constant. These risks are as great as any other occupational hazards affecting surgeons today. The time has come to recognize and address them. 